Mathematical Modelling and Analysis

Volume 17 Number 5, November 2012, 618-629 http://dx.doi.org/10.3846/13926292.2012.732972

(c) Vilnius Gediminas Technical University, 2012
Publisher: Taylor\&Francis and VGTU

http://www.tandfonline.com/TMMA

Print ISSN: 1392-6292

Online ISSN: 1648-3510

\title{
Dual Equation and Inverse Problem for an Indefinite Sturm-Liouville Problem with $m$ Turning Points of Even Order
}

\author{
Hamidreza Marasi $^{a}$ and Aliasghar Jodayree Akbarfam $^{b}$ \\ ${ }^{a}$ University of Bonab \\ Bonab, Iran \\ ${ }^{b}$ University of Tabriz \\ Tabriz, Iran \\ E-mail: marasi@bonabu.ac.ir
}

Received September 11, 2011; revised September 5, 2012; published online November 1, 2012

\begin{abstract}
In this paper the differential equation $y^{\prime \prime}+\left(\rho^{2} \phi^{2}(x)-q(x)\right) y=0$ is considered on a finite interval $\mathrm{I}$, say $I=[0,1]$, where $q$ is a positive sufficiently smooth function and $\rho^{2}$ is a real parameter. Also, $[0,1]$ contains a finite number of zeros of $\phi^{2}$, the so called turning points, $0<x_{1}<x_{2}<\cdots<x_{m}<1$. First we obtain the infinite product representation of the solution. The product representation, satisfies in the original equation. As a result the associated dual equation is derived and then we proceed with the solution of the inverse problem.
\end{abstract}

Keywords: turning point, Sturm-Liouville problem, indefinite problem, dual equation, inverse problem.

AMS Subject Classification: 34E55; 34B05; 34E20; 34L05; $47 \mathrm{E} 05$.

\section{Introduction}

In most differential equations with variable coefficients it is impossible to obtain an exact solution, so one must resort to various approximation methods of solution. One of the most useful mathematical methods of achieving this, is representing the solution by an asymptotic form $[15,16]$. But, in methods connected with dual equations, the closed form of the solution is needed. For this purpose we know from Halvorsen result [6], that any solution $y(x, \lambda)$ of the following equation

$$
y^{\prime \prime}+\left(\lambda \phi^{2}(x)-q(x)\right) y=0, \quad x \in I=[0,1],
$$

defined by a fixed set of initial conditions is an entire function of $\lambda$ for each fixed $x \in[0,1]$, whose order does not exceed $\frac{1}{2}$. Thus it follows from the classical Hadamard factorization theorem (see [12]), such solutions are expressible as an infinite product. 
In physics, engineering and quantum mechanics it is necessary to build a mathematical model to represent certain problems, e.g. the determining of the inter-atomic forces for given energy-levels. This leads to the following general problem.

Given the spectrum of a second-order differential operator, to determine this operator.

This is known as the inverse problem. It may be pointed out that the inverse problem may not be properly formulated. Marchenko in [17] has shown that the problem can only have a solution if the spectral distribution function $\sigma(\lambda)$ of the operator is given. The spectral distribution function is defined in term of the normalization constants of the eigenfunctions. Levitan and Gasymov in [13] gave a new account of the solution of the inverse problem in terms of the spectral function in the case a classical Sturm-Liouville operator. The inverse problem for the canonical case of a two-term Sturm-Liouville operator on a finite interval is completely solved in [21]. In [21] the authors introduced the Dirichlet spectrum associated with the function $q$ in $L^{2}$ and derived some of its properties. Belishev in [14] considered the homogeneous boundary-value problem

$$
y^{\prime \prime}+\lambda p(x) y=0, \quad x \in[0, l](l<\infty)
$$

with the conditions $y(0)=y(l)=0$. The density $p(x)$ is real-valued, $p(x) \in$ $L_{1}(0, l), p(x) \neq 0$ almost everywhere on $(0, l)$. Let $\sigma=\left\{\lambda_{j}\right\}_{-\infty}^{\infty}$ be the negative and positive eigenvalues of the problem (1.2) and $\left\{Q_{j}(x)\right\}_{-\infty}^{\infty}$ be the corresponding eigenfunctions which are normalized by the condition $Q_{j}(0)=1$ for all $j$. In [14] it is shown that the function $p(x)$ can be reconstructed from the spectrum $\sigma$ and a knowledge of $\epsilon=\left\{v_{j}\right\}_{-\infty}^{\infty}$ where $v_{j}=\int_{0}^{l}\left[Q_{j}^{\prime}(x)\right]^{2} d x$. In [20] Pranger considered differential equation (1.2) in which $p(x)$ is a positive twice continuously differentiable function on $[0,1]$. In this article he studied the recovery of the function $p(x)$ from the knowledge of the eigenvalues. Differential equations with turning points have various applications in mathematics, elasticity, optics, geophysics and other branches of natural sciences (see $[1,2,5,18,22]$ and the references therein). Inverse spectral theory for indefinite Sturm-Liouville operators is not as well developed as the corresponding theory for definite cases. Indeed, there are very few papers for example $[3,8,9,11]$, all dealing with case of low number of turning points and certainly pertaining only to given real spectral data as opposed to cases where non-real spectra may occur (see [19]). In [10] authors considered the following Sturm-Liouville equation

$$
y^{\prime \prime}+\left(\lambda \phi^{2}(x)-q(x)\right) y=0, \quad-1 \leq x \leq 1 .
$$

It is assumed that $q(x)$ is a real function that is Lebesgue integrable on the interval $[-1,1], \lambda=\rho^{2}$ is the spectral parameter and

$$
\phi^{2}(x)=\left(x-x_{1}\right)^{4 m+1} \phi_{0}(x),
$$

where $-1<x_{1}<1, m \in N, \phi_{0}>0$ for $x \in[-1,1]$, $\phi_{0}$ is a twice continuously differentiable on $[0,1]$ and $\phi^{2}(x)$ has one zero in $[-1,1]$, so called turning point. In [10] a unique positive potential has reconstructed from two spectral 
sequences, given a weight function with a simple turning point in the interior of a finite interval with fixed end boundary conditions. In this paper we proceed to solve such inverse problem in a case where the weight function (the coefficient of $\lambda$ in (1.3)) has $m$ turning points that one of odd order and others are of even order.

\section{Notations and Preliminary Results}

Let us consider the real second order differential equation

$$
y^{\prime \prime}+\left(\rho^{2} \phi^{2}(x)-q(x)\right) y=0, \quad x \in I=[0,1]
$$

where $\rho^{2}=\lambda$ is the spectral parameter, $q$ is bounded and integrable on $I$ and

$$
\phi^{2}(x)=\phi_{0}(x) \prod_{v=1}^{m}\left(x-x_{v}\right)^{\ell_{v}},
$$

where $0=x_{0}<x_{1}<x_{2}<\cdots<x_{m}<1=x_{m+1}, \phi_{0}(x)>0$ for $x \in I=[0,1]$ and $\phi_{0}$ is twice continuously differentiable on $I$. In other words, $\phi^{2}(x)$ has in $I$ $m$ zeros $x_{v}, v=1, \ldots, m$ of order $l_{v}$, so called turning points.

We distinguish four different types of turnings points: For $1 \leq v \leq m$

$$
T_{v}= \begin{cases}I, & \text { if } l_{v} \text { is even and } \phi^{2}(x)\left(x-x_{v}\right)^{-l_{v}}<0 \\ I I, & \text { if } l_{v} \text { is even and } \phi^{2}(x)\left(x-x_{v}\right)^{-l_{v}}>0, \\ I I I, & \text { if } l_{v} \text { is odd and } \phi^{2}(x)\left(x-x_{v}\right)^{-l_{v}}<0, \\ I V, & \text { if } l_{v} \text { is odd and } \phi^{2}(x)\left(x-x_{v}\right)^{-l_{v}}>0\end{cases}
$$

is called type of $x_{v}$.

Specially in this paper, we suppose that $\ell_{1}=4 k+1$ and $\ell_{v}=4 k$, $v=2,3, \ldots, m$. In other words, $x_{1}$ is of type $I V$ while $x_{2}, x_{3}, \ldots, x_{m}$ are of type $I I$.

In order to represent the solution as an infinite product and then study the inverse problem we use a suitable fundamental system of solutions (FSS) for equation (2.1) as provided in [2]. Let $\epsilon>0$ be fixed, sufficiently small and $D_{0 \epsilon}=\left[0, x_{1}-\epsilon\right], D_{v \epsilon}=\left[x_{v}+\epsilon, x_{v+1}-\epsilon\right]$ for $1 \leq v \leq m-1, D_{m \epsilon}=\left[x_{m}+\epsilon, 1\right]$, $D_{\epsilon}=\bigcup_{v=0}^{m} D_{v \epsilon}$ and $I_{v \epsilon}=D_{v-1, \epsilon} \cup\left[x_{v}-\epsilon, x_{v}+\epsilon\right] \cup D_{v \epsilon}$.

Further we set for $1 \leq v \leq m$

$$
\mu_{v}=\frac{1}{2+l_{v}}, \quad \theta_{v}= \begin{cases}1, & \mu_{v}>\frac{1}{4} \\ 1-\delta_{0}, & \mu_{v}=\frac{1}{4} \\ 4 \mu_{v}, & \mu_{v}<\frac{1}{4}\end{cases}
$$

and $0<\theta_{0}=\min \left\{\theta_{v} \mid 1 \leq v \leq m\right\}$. We also denote

$$
\begin{aligned}
& I_{+}=\left\{x \mid \phi^{2}(x)>0\right\}, \quad I_{-}=\left\{x \mid \phi^{2}(x)<0\right\}, \quad \xi(x)= \begin{cases}0, & x \in I_{+}, \\
1, & x \in I_{-},\end{cases} \\
& \phi_{+}^{2}(x)=\max \left(0, \phi^{2}(x)\right), \quad \phi_{-}^{2}(x)=\max \left(0,-\phi^{2}(x)\right),
\end{aligned}
$$




$$
\begin{aligned}
& \gamma_{v}=\left\{\begin{array}{ll}
2 \sin \frac{\pi \mu_{v}}{2}, & T_{v}=I I, I V, \\
\sin \pi \mu_{v}, & T_{v}=I, I I,
\end{array} \vartheta_{v}= \begin{cases}2, & T_{v}=I I I, I V, \\
1, & T_{v}=I, I I,\end{cases} \right. \\
& K_{ \pm}(x)=\left(\prod_{x_{v} \in(0, x)} \gamma_{v}^{-1}\right) e^{ \pm \iota \frac{\pi}{4}(\xi(x)-\xi(0))} \\
& K_{ \pm}^{*}(x)=\left(\prod_{x_{v} \in(0, x)} \gamma_{v}^{-1}\right) e^{ \pm \iota \frac{\pi}{4}(\xi(x)+\xi(0))} .
\end{aligned}
$$

Clearly,

$$
K_{ \pm}(x) K_{ \pm}^{*}(x)=e^{ \pm \iota \frac{\pi}{2} \xi(x)}= \begin{cases}1, & x \in I_{+} \\ \pm \iota, & x \in I_{-}\end{cases}
$$

Let

$$
\begin{aligned}
& S_{k}=\left\{\rho: \arg \rho \in\left[\frac{k \pi}{4}, \frac{(k+1) \pi}{4}\right]\right\}, \\
& \sigma_{s}^{\delta}=\left\{\rho: \arg \rho \in\left[\frac{s \pi}{2}-\delta, \frac{s \pi}{2}+\delta\right]\right\}, \quad \delta>0, \\
& \sigma^{\delta}=\bigcup_{s} \sigma_{s}^{\delta}, \quad S_{k}^{\delta}=S_{k}-\sigma^{\delta}, \quad S^{\delta}=\bigcup_{k=-2}^{1} S_{k}^{\delta} .
\end{aligned}
$$

It is sufficient to consider the sectors $S_{k}$ and $S_{k}^{\delta}$ for $k=-2,-1,0,1$ only.

It is shown in [2] that for each fixed sector $S_{k}(k=-2,-1,0,1)$ there exist a fundamental system of solutions of (2.1) $\left\{Z_{1}(x, \rho), Z_{2}(x, \rho)\right\}, x \in I, \rho \in S_{k}$ such that the functions

$$
(x, \rho) \mapsto Z_{j}^{s}(x, \rho) \quad(j=1,2 ; s=0,1)
$$

are continues for $x \in I, \rho \in S_{k}$ and holomorphic for each fixed $x \in I$ with respect to $\rho \in S_{k}$, moreover for $|\rho| \rightarrow \infty, \rho \in S_{k}, x \in D_{\epsilon}, j=1,2$.

$$
\begin{aligned}
Z_{1}^{(j)}(x, \rho)= & ( \pm \iota \rho)^{j}|\phi(x)|^{j-\frac{1}{2}}\left(\exp \left(\mp \iota \frac{\pi}{2} \xi(x)\right)^{j} \exp \left(\rho \int_{o}^{x}\left|\phi_{-}(t)\right| d t\right)\right. \\
& \times \exp \left( \pm \int_{0}^{x}\left|\phi_{+}(t)\right| d t\right) K_{ \pm}(x) \kappa(x, \rho), \\
Z_{2}^{(j)}(x, \rho)= & (\mp \iota \rho)^{j}|\phi(x)|^{j-\frac{1}{2}}\left(\exp \left(\mp \iota \frac{\pi}{2} \xi(x)\right)^{j} \exp \left(-\rho \int_{o}^{x}\left|\phi_{-}(t)\right| d t\right)\right. \\
& \times \exp \left(\mp \int_{0}^{x}\left|\phi_{+}(t)\right| d t\right) K_{ \pm}^{*}(x) \kappa(x, \rho), \\
W(\rho)= & W\left(Z_{1}(x, \rho), Z_{2}(x, \rho)\right)=\mp(2 \iota \rho)[1],
\end{aligned}
$$

where $W(f(x), g(x)):=f(x) g^{\prime}(x)-f^{\prime}(x) g(x)$ is the Wronskian of $f$ and $g$. Note that, the choice of the root $\phi(x)$ of $\phi^{2}(x)$ depends on the interval and has to determined carefully. For example, in this paper, due to the type of turning points $x_{1}$ and $x_{v}, v=2,3, \ldots, m$ we have for $\rho \in S_{0}$

$$
\phi(x)= \begin{cases}|\phi(x)|, & x>x_{1}, \\ |\phi(x)| e^{i \pi / 2}, & x<x_{1} .\end{cases}
$$




\section{Dual Equation}

Let $S(x, \lambda)$ solve the second order differential equation (2.1) with initial conditions

$$
S(0, \lambda)=0, \quad S^{\prime}(0, \lambda)=1 .
$$

For $x \in\left(0, x_{1}\right)$, the Dirichlet problem associated (2.1) in interval [0,x] (Eq. (2.1) with boundary conditions $y(0, \lambda)=0=y(x, \lambda)=0$ ) has a countable set of negative eigenvalues, $\left\{\lambda_{n}(x)\right\}_{n=1}^{\infty}$, with the following asymptotic distribution

$$
\sqrt{-\lambda_{n}(x)}=\frac{n \pi}{\int_{0}^{x}|\phi(t)| d t}+O\left(\frac{1}{n}\right) .
$$

In this case, the condition $S\left(x, \lambda_{n}(x)\right)=0$ gives, as usual,

$$
\frac{\partial S}{\partial x}+\frac{\partial S}{\partial \lambda} \lambda_{n}^{\prime}=0
$$

and differentiating again

$$
\frac{\partial^{2} S}{\partial x^{2}}+2 \frac{\partial^{2} S}{\partial x \partial \lambda} \lambda_{n}^{\prime}+\frac{\partial^{2} S}{\partial \lambda^{2}}\left(\lambda_{n}^{\prime}\right)^{2}+\frac{\partial S}{\partial \lambda} \lambda_{n}^{\prime \prime}=0
$$

The first term in (3.1) is zero at $\left(x, \lambda_{n}(x)\right)$ by (2.1). So we have

$$
2 \frac{\partial^{2} S}{\partial x \partial \lambda} \lambda_{n}^{\prime}+\frac{\partial^{2} S}{\partial \lambda^{2}}\left(\lambda_{n}^{\prime}\right)^{2}+\frac{\partial S}{\partial \lambda} \lambda_{n}^{\prime \prime}=0 .
$$

For $x \in\left(x_{v}, x_{v+1}\right), v=1,2, \ldots, m$, the spectrum $\left\{\lambda_{n}\right\}$ of the similar Dirichlet boundary value problem consist of two sequences of negative, $u_{n v}(x)$, and positive, $r_{n v}(x)$, eigenvalues: $\left\{\lambda_{n}(x)\right\}=\left\{u_{n v}(x)\right\} \cup\left\{r_{n v}(x)\right\}, n \in N$, such that

$$
\begin{aligned}
\sqrt{u_{n v}(x)} & =\frac{n \pi-\pi / 4}{\int_{x_{1}}^{x}|\phi(t)| d t}+O\left(\frac{1}{n}\right), \\
\sqrt{-r_{n v}(x)} & =-\frac{n \pi-\pi / 4}{\int_{0}^{x_{1}}|\phi(t)| d t}+O\left(\frac{1}{n}\right) .
\end{aligned}
$$

Also, from the conditions

$$
S\left(x, u_{n v}(x)\right)=0, \quad S\left(x, r_{n v}(x)\right)=0,
$$

we have

$$
\begin{aligned}
& 2 \frac{\partial^{2} S}{\partial x \partial \lambda} u_{n v}^{\prime}+\frac{\partial^{2} S}{\partial \lambda^{2}}\left(u_{n v}^{\prime}\right)^{2}+\frac{\partial S}{\partial \lambda} u_{n v}^{\prime \prime}=0 \\
& 2 \frac{\partial^{2} S}{\partial x \partial \lambda} r_{n v}^{\prime}+\frac{\partial^{2} S}{\partial \lambda^{2}}\left(r_{n v}^{\prime}\right)^{2}+\frac{\partial S}{\partial \lambda} r_{n v}^{\prime \prime}=0
\end{aligned}
$$

Now, since by Halvorsen's result [6], the solution $S(x, \lambda)$ is an entire function of $\lambda$ for each fixed $x \in[0,1]$, thus it follows from the classical Hadamard's factorization theorem (see [12, p. 24]) that such solution is expressible as an infinite product. 
For fixed $x \in\left(0, x_{1}\right)$ we have (see [16])

$$
S(x, \lambda)=\frac{1}{2 \rho}|\phi(x) \phi(0)|^{-\frac{1}{2}} e^{\rho \int_{0}^{x} \phi(t) d t} E_{k}(x, \rho)=h(x) \prod_{n=1}^{\infty}\left(1-\frac{\lambda}{\lambda_{n}(x)}\right),
$$

where $h(x)$ is a function independent of $\lambda$ but may depend on $x$ and

$$
h_{1}(x)=|\phi(x) \phi(0)|^{-\frac{1}{2}} R_{-}(x)=h(x) \prod \frac{-z_{k}^{2}}{\lambda_{k}}
$$

also $R_{-}(x)=\int_{0}^{x} \sqrt{\max \left\{0,-\phi^{2}(t)\right\}} d t$ and $z_{k}=\frac{k \pi}{R_{-}(x)}$. Then

$$
h(x)=|\phi(x) \phi(0)|^{-\frac{1}{2}} R_{-}(x) \prod \frac{-\lambda_{k}}{z_{k}^{2}} .
$$

Similarly, for the case $x_{v}<x<x_{v+1}$ we have

$$
S(x, \lambda)=g(x) \prod_{n \geq 1}\left(1-\frac{\lambda}{r_{n v}(x)}\right)\left(1-\frac{\lambda}{u_{n v}(x)}\right)
$$

with

$$
\begin{aligned}
g(x)= & \frac{\pi}{8}|\varphi(x)|^{-\frac{1}{2}}|\varphi(0)|^{\frac{1}{2}}\left(R_{-}\left(x_{1}\right) R_{+}(x)\right)^{\frac{1}{2}} \csc \frac{\pi \mu_{1}}{2} \csc \pi \mu_{2} \cdots \csc \pi \mu_{v} \\
& \times \prod \frac{R_{+}^{2}(x) \lambda_{n}^{+}(x)}{\tilde{j}_{k}^{2}} \prod \frac{R_{-}^{2}\left(x_{1}\right) \lambda_{n}^{-}(x)}{\tilde{j}_{k}^{2}} .
\end{aligned}
$$

Now if we make use of the above infinite product forms of $S(x, \rho),(3.4)$ for $0<x<x_{1}$, substitute in (3.2) and (3.6) for $x_{v}<x<x_{v+1}$, substitute in (3.3) we will obtain Dual equation of (2.1). Indeed we need the various derivatives of $S(x, \rho)$ at the points $\left(x, \lambda_{n}(x)\right)$ for $0<x<x_{1}$ and at the points $\left(x, u_{n v}(x)\right)$ and $\left(x, r_{n v}(x)\right)$ for $x_{v}<x<x_{v+1}$. We calculate $\frac{\partial S}{\partial \lambda}, \frac{\partial^{2} S}{\partial \lambda^{2}}$ and $\frac{\partial^{2} S}{\partial x \partial \lambda}$ at the points $\left(x, \lambda_{n}(x)\right)$ by using (3.4). In forming $\frac{\partial^{2} S}{\partial x \partial \lambda}$ the interchange of summation and differentiation in

$$
\frac{d}{d x} \sum \log \left(1-\frac{\lambda}{\lambda_{k}(x)}\right)
$$

will be valid if the differentiated series

$$
\sum_{k \neq n} \frac{-\lambda_{n} \lambda_{k}^{\prime}(x)}{\left(\lambda_{k}(x)-\lambda_{n}\right) \lambda_{k}(x)}
$$

is uniformly convergent. According to [7, Lemma 4.21] the above series is uniformly convergent. We define $F_{n}$ by

$$
F_{n}=F_{n}\left(x, \lambda_{n}(x)\right)=\prod_{k \neq n, 1 \leq k}\left(1-\frac{\lambda_{n}(x)}{\lambda_{k}(x)}\right) .
$$

Since

$$
\frac{\partial S}{\partial \lambda}=h \sum_{i=1}^{\infty} \frac{-1}{\lambda_{i}(x)} \prod_{k \neq n, 1 \leq k}\left(1-\frac{\lambda}{\lambda_{k}(x)}\right)
$$


we have

$$
\begin{aligned}
& \frac{\partial S}{\partial \lambda}\left(x, \lambda_{n}\right)=\frac{-h F_{n}}{\lambda_{n}(x)} \\
& \frac{\partial^{2} S}{\partial \lambda^{2}}\left(x, \lambda_{n}(x)\right)=\frac{2 h F_{n}}{\lambda_{n}(x)} \sum_{i \neq n, 1 \leq i} \frac{1}{\lambda_{i}}\left(1-\frac{\lambda_{n}(x)}{\lambda_{i}(x)}\right)^{-1} \\
& \frac{\partial^{2} S}{\partial \lambda \partial x}=\frac{-h^{\prime}(x) F_{n}}{\lambda_{n}(x)}+\frac{h(x) \lambda_{n}^{\prime} F_{n}}{\lambda_{n}^{2}}-h(x) F_{n} \sum_{i \neq n, 1 \leq i} \frac{\lambda_{i}^{\prime}}{\lambda_{i}^{2}}\left(1-\frac{\lambda_{n}(x)}{\lambda_{i}(x)}\right)^{-1} \\
& \quad-\frac{h(x) \lambda_{n}^{\prime} F_{n}}{\lambda_{n}} \sum_{i \neq n, 1 \leq i} \frac{1}{\lambda_{i}}\left(1-\frac{\lambda_{n}(x)}{\lambda_{i}(x)}\right)^{-1} .
\end{aligned}
$$

Placing these terms in to (3.2) we obtain

$$
\lambda_{n}^{\prime \prime}+\frac{2 h^{\prime} \lambda_{n}^{\prime}}{h}+2 \lambda \lambda_{n}^{\prime} \sum_{i \neq n, 1 \leq i} \frac{\lambda_{i}^{\prime}}{\lambda_{i}^{2}}\left(1-\frac{\lambda_{n}(x)}{\lambda_{i}(x)}\right)^{-1}-2 \frac{\left(\lambda_{n}^{\prime}\right)^{2}}{\lambda_{n}}=0 .
$$

Dividing the above equation by $\lambda_{n}^{\prime}$ and integrating from a fixed point $\alpha \neq 0$ up to $x, 0<x<x_{1}$, we obtain

$$
\lambda_{n}^{\prime}(x)=\frac{\lambda_{n}^{2}(x) \lambda_{n}^{\prime}(\alpha) h^{2}(\alpha)}{\lambda_{n}^{2}(\alpha) h^{2}(x)} e^{-2 S_{n}\left(x, \lambda_{n}\right)},
$$

where

$$
S_{n}\left(x, \lambda_{n}\right)=\sum_{i \neq n} \int_{\alpha}^{x} \frac{\lambda_{i}^{\prime} \lambda_{n}}{\lambda_{i}}\left(\lambda_{i}-\lambda_{n}\right)^{-1},
$$

and $h(x)$ is determined in (3.5).

As before, for the case $x_{v}<x<x_{v+1}$, we calculate the various derivatives of $S(x, \rho)$ and evaluate these at the fixed points $\left(x, u_{n v}(x)\right)$. Since, similarly, the series

$$
\sum_{k \neq n} \frac{-u_{n v} u_{k v}^{\prime}(x)}{\left(u_{k v}(x)-u_{n v}\right) u_{k v}(x)}
$$

is uniformly convergent, we obtain $\frac{\partial^{2} S}{\partial \lambda \partial x}$ from (3.6) in terms of $u_{n v}$ and $r_{n v}$. Suppose

$$
G_{n}(x, \lambda)=\prod_{k \neq n, 1 \leq k}\left(1-\frac{\lambda}{u_{k v}(x)}\right), \quad H_{n}(x, \lambda)=\prod_{1 \leq k}\left(1-\frac{\lambda}{r_{k v}(x)}\right) .
$$

Then

$$
\begin{aligned}
& G_{n}=G_{n}\left(x, u_{n v}(x)\right)=\prod_{k \neq n, 1 \leq k}\left(1-\frac{u_{n v}(x)}{u_{k v}(x)}\right), \\
& H_{n}=H_{n}\left(x, u_{n v}(x)\right)=\prod_{1 \leq k}\left(1-\frac{u_{n v}(x)}{r_{k v}(x)}\right),
\end{aligned}
$$


so that

$$
\prod_{k \neq i, 1 \leq k}\left(1-\frac{u_{n v}(x)}{r_{k v}(x)}\right)=H_{n}\left(1-\frac{u_{n v}}{r_{i v}}\right)^{-1}
$$

We have

$$
\begin{aligned}
& \frac{\partial S}{\partial \lambda}\left(x, u_{n v}\right)=\frac{-g(x) H_{n} G_{n}}{u_{n v}(x)}, \\
& \frac{\partial^{2} S}{\partial \lambda^{2}}\left(x, u_{n v}(x)\right)=\frac{2 g H_{n} G_{n}}{u_{n v}(x)} \sum_{1 \leq i} \frac{1}{r_{i v}(x)-u_{n v}(x)} \\
& \quad+\frac{2 g H_{n} G_{n}}{u_{n v}(x)} \sum_{1 \leq i, i \neq n} \frac{1}{u_{i v}(x)-u_{n v}(x)}, \\
& \frac{\partial^{2} S}{\partial \lambda \partial x}\left(x, u_{n v}(x)\right)=\frac{-g^{\prime}(x) H_{n} G_{n}}{u_{n v}(x)}+\frac{g(x) H_{n} G_{n} u_{n v}^{\prime}}{u_{n v}}\left(\frac{1}{u_{n v}}\right. \\
& \left.\quad-\sum_{1 \leq i} \frac{1}{r_{i v}(x)-u_{n v}(x)}-\sum_{1 \leq i, i \neq n} \frac{1}{u_{i v}(x)-u_{n v}(x)}\right) \\
& \quad-g(x) H_{n} G_{n}\left(\sum_{1 \leq i} \frac{r_{i v}^{\prime}}{r_{i v}}\left(r_{i v}(x)-u_{n v}(x)\right)^{-1}-\sum_{1 \leq i, i \neq n} \frac{u_{i v}^{\prime}}{u_{i v}}\left(u_{i v}(x)-u_{n v}(x)\right)^{-1}\right) .
\end{aligned}
$$

Placing these terms into (3.3) we obtain

$$
\begin{aligned}
& u_{n v}^{\prime \prime}+\frac{2 g^{\prime} u_{n v}^{\prime}}{g}+2 u_{n v} u_{n v}^{\prime}\left\{\sum_{1 \leq i, i \neq n} \frac{u_{i v}^{\prime}}{u_{i v}}\left(u_{i v}(x)-u_{n v}(x)\right)^{-1}\right. \\
& \left.+\sum_{1 \leq i} \frac{r_{i v}^{\prime}}{r_{i v}}\left(r_{i v}(x)-u_{n v}(x)\right)^{-1}\right\}-2 \frac{\left(u_{n v}^{\prime}\right)^{2}}{u_{n v}}=0 .
\end{aligned}
$$

Similarly for negative eigenvalues, $r_{n v}(x)$ we get

$$
\begin{aligned}
& r_{n v}^{\prime \prime}+\frac{2 g^{\prime} r_{n v}^{\prime}}{g}+2 r_{n v} r_{n v}^{\prime}\left\{\sum_{1 \leq i, i \neq n} \frac{r_{i v}^{\prime}}{r_{i v}}\left(r_{i v}(x)-r_{n v}(x)\right)^{-1}\right. \\
& \left.+\sum_{1 \leq i} \frac{u_{i v}^{\prime}}{u_{i v}}\left(u_{i v}(x)-r_{n v}(x)\right)^{-1}\right\}-2 \frac{\left(r_{n v}^{\prime}\right)^{2}}{r_{n v}}=0 .
\end{aligned}
$$

Dividing the equation (3.9) by $u_{n v}^{\prime}$, the equation (3.10) by $r_{n v}^{\prime}$ and integrating from $x, x_{v}<x<x_{v+1}$, up to $\alpha \neq x_{v+1}$, we deduce

$$
\begin{aligned}
& u_{n v}^{\prime}(x)=\frac{u_{n v}^{2}(x) u_{n v}^{\prime}(\alpha) g^{2}(\alpha)}{u_{n v}^{2}(\alpha) g^{2}(x)} e^{2 T_{n}\left(x, u_{n v}, r_{n v}\right)}, \\
& r_{n v}^{\prime}(x)=\frac{r_{n v}^{2}(x) r_{n v}^{\prime}(\alpha) g^{2}(\alpha)}{r_{n v}^{2}(\alpha) g^{2}(x)} e^{2 T_{n}\left(x, u_{n v}, r_{n v}\right)},
\end{aligned}
$$

where

$$
T_{n}\left(x, u_{n v}, r_{n v}\right)=\sum_{i \neq n} \int_{x}^{\alpha} \frac{u_{i v}^{\prime} u_{n v}}{u_{i v}}\left(u_{i v}-u_{n v}\right)^{-1} d v+\sum_{i} \int_{x}^{\alpha} \frac{r_{i v}^{\prime} u_{n v}}{r_{i v}}\left(r_{i v}-u_{n v}\right)^{-1} d v
$$


$g(x)$ is determined in (3.7) and the variables in (3.12) are dropped for brevity. The system of equations (3.8) and (3.11) is dual to original equation (2.1) and involves only the functions $\lambda_{n}(x), u_{n v}(x)$ and $r_{n v}(x)$.

\section{Inverse Problem}

We now proceed with the solution of the inverse problem. We study the relation between the eigenvalues and the potential function.

Theorem 1. Let $\lambda_{n}(x)$ be the eigenvalues of the Dirichlet problem (2.1) on $[0, x]$, for fixed $0<x<x_{1}$. Then

$$
\begin{aligned}
& \text { a) } \sigma_{1}(x)=\sum_{k=1}^{\infty} \frac{1}{\lambda_{n}(x)}=\int_{0}^{x} \frac{1}{h^{2}(t)} \int_{0}^{t} \phi^{2}(v) h^{2}(v) d v d t \\
& \text { b) } q(x)=\left(\frac{\phi(x)-\sigma_{1}^{\prime \prime}}{2 \sigma_{1}^{\prime}}\right)^{2}+\left(\frac{\phi(x)-\sigma_{1}^{\prime \prime}}{2 \sigma_{1}^{\prime}}\right)^{\prime},
\end{aligned}
$$

where $h(x)$ is determined in (3.5).

Proof. a) For fixed $0<x<x_{1}$, if $S(x, \lambda)$ in (3.4) is logarithmical differentiated, then

$$
-\frac{\partial}{\partial \lambda} \log S(x, \lambda)=\sum_{k=1}^{\infty} \frac{1}{\lambda_{k}-\lambda}=\sum_{k=1}^{\infty} \sum_{m=0}^{\infty} \frac{\lambda^{m}}{\lambda_{k}^{m+1}},
$$

provided that $|\lambda|<\left|\lambda_{1}\right|$. The last series is absolutely convergent because

$$
\sum \frac{1}{\left|\lambda_{k}-\lambda\right|} \leq \sum \frac{1}{\left|\lambda_{k}\right|-|\lambda|}=\sum O\left(\frac{1}{k^{2}}\right),
$$

thus we can interchange the order of summation. Put

$$
\sum_{k=1}^{\infty} \frac{1}{\lambda_{k}^{m+1}}=\sigma_{m+1}
$$

and interchange the order of summation. Then

$$
-\frac{\partial}{\partial \lambda} S(x, \lambda)=S(x, \lambda) \sum_{m=0}^{\infty} \sigma_{m+1} \lambda^{m} .
$$

Replace $S(x, \lambda)$ on each side by

$$
S(x, \lambda)=a_{0}(x)+a_{1}(x) \lambda+a_{2}(x) \lambda^{2}+\cdots .
$$

Multiply out the product on the right and equate coefficients of the various powers of $\lambda$ in the identity. Thus we obtain the system of equations

$$
\begin{aligned}
-a_{1}(x) & =a_{0}(x) \sigma_{1} \\
-2 a_{2}(x) & =a_{0}(x) \sigma_{2}+a_{(}(x) \sigma_{1} \\
& \ldots \\
-n a_{n}(x) & =a_{0}(x) \sigma_{n}+a_{1}(x) \sigma_{n-1}+\cdots+a_{n-1}(x) \sigma_{1} .
\end{aligned}
$$


From (3.4) and (4.3) we have $S(x, 0)=h(x)=a_{0}(x)$. Using the expansion of $S(x, \lambda)$ in $(2.1)$ we get

$$
a_{0}^{\prime \prime}+\lambda_{1}^{\prime \prime}+\cdots+\left(\lambda \phi^{2}(x)-q(x)\right)\left(a_{0}+a_{1} \lambda+\cdots\right)=0 .
$$

Consequently, by equating coefficients of the various powers of $\lambda$ we obtain

$$
\begin{aligned}
a_{0}^{\prime \prime}-q a_{0} & =0 \\
a_{1}^{\prime \prime}+\phi^{2}(x) a_{0}-a_{1} q(x) & =0 \\
a_{2}^{\prime \prime}+\phi^{2}(x) a_{1}-a_{2} q(x) & =0
\end{aligned}
$$

Placing (4.4) in (4.5) and using $a_{0}^{\prime \prime}=q a_{0}$ we deduce

$$
a_{0}^{2} \sigma_{1}^{\prime \prime}+2 a_{0} a_{0}^{\prime} \sigma_{1}^{\prime}-\phi^{2}(x) a_{0}^{2}=0,
$$

or

$$
\frac{d}{d x}\left(a_{0}^{2} \sigma_{1}^{\prime}\right)=\phi^{2}(x) a_{0}^{2} .
$$

Integrating this over $[0, x]$, nothing that $a_{i}(0)=0$, we see that, after another integration and use of the fact that $\sigma_{1}(0)=0$ and $a_{0}=h,(4.1)$ holds.

b) From (4.6) we write

$$
\sigma_{1}^{\prime \prime}+2 \frac{a_{0}^{\prime}}{a_{0}} \sigma_{1}^{\prime}-\phi^{2}(x)=0 .
$$

Consequently

$$
\left(\frac{a_{0}^{\prime}}{a_{0}}\right)^{\prime}=\left(\frac{\phi^{2}(x)-\sigma_{1}^{\prime \prime}}{2 \sigma_{1}^{\prime}}\right)^{\prime},
$$

whence

$$
\begin{aligned}
q(x) & =\frac{a_{0}^{\prime \prime}}{a_{0}}=\left(\frac{a_{0}^{\prime}}{a_{0}}\right)^{\prime}+\left(\frac{a_{0}^{\prime}}{a_{0}}\right)^{2} \\
& =\left(\frac{\phi^{2}(x)-\sigma_{1}^{\prime \prime}}{2 \sigma_{1}^{\prime}}\right)^{\prime}+\left(\frac{\phi^{2}(x)-\sigma_{1}^{\prime \prime}}{2 \sigma_{1}^{\prime}}\right)^{2}, \quad 0<x<x_{1} .
\end{aligned}
$$

Applying arguments similar to above, we deduce the following theorem for $x_{v}<x<x_{v+1}$.

Theorem 2. Let the sequence $u_{n v}(x)$ represent the positive eigenvalues and $r_{n v}(x)$ the negative eigenvalues of the Dirichlet problem $(2.1)$ on $[0, x]$ for fixed $x_{v}<x<x_{v+1}, v=1,2, \ldots, m$. Then

$$
\text { a) } \begin{aligned}
\Lambda_{1}(x) & =\sum_{k=1}^{\infty}\left[\frac{1}{u_{k v}(x)}+\frac{1}{r_{k v}(x)}\right] \\
& =\Lambda_{1}\left(x_{v+1}\right)-\int_{x}^{x_{v+1}} \frac{1}{g^{2}(t)} \int_{x_{v}}^{t} \phi^{2}(v) g^{2}(v) d v d t, \\
\text { b) } q(x) & =\left(\frac{\phi^{2}(x)-\Lambda_{1}^{\prime \prime}}{2 \Lambda_{1}^{\prime}}\right)^{2}+\left(\frac{\phi^{2}(x)-\Lambda_{1}^{\prime \prime}}{2 \Lambda_{1}^{\prime}}\right),
\end{aligned}
$$

where $g(x)$ is determined in (3.7). 


\section{Conclusions}

In recent years there appeared many new areas for applications of inverse Sturm-Liouville problems. For example, discontinuous inverse problems appear in electronics for constructing parameters of heterogeneous electronic lines with desirable technical characteristics. In most papers the inverse problem was studied in the case that the weight function $\phi^{2}(x)$ does not change sign. But many further applications connect with differential equation of the form (2.1) with turning points. For example, turning points connect with physical situations in which zeros correspond to the limit of motion of a wave mechanical particle bound by a potential field [4]. In this paper, the weight function changes sign and has zeros. The potential function was obtained in terms of trace functions $\sigma_{1}(x)$ and $\Lambda_{1}(x)$ which are defined in (4.1) and (4.7) respectively. On the other hand in one turning point case, the solution can be obtained by using conditions of the problem, without any additional effort. Here, in the case of multiple turning points, we should apply matching process for obtaining the solution, infinite product of the solution and solving $m+1$ dual equations. In fact, beginning from the last interval, in interval $D_{m, \epsilon}$ the set of positive and negative eigenvalues $\left\{u_{n, m}\right\}_{n=1}^{\infty} \cup\left\{r_{n, m}\right\}_{n=1}^{\infty}$ can be obtained by solving the corresponding dual equations (3.11) with the given spectrum $u_{n, m}(1)=u_{n}$ and $r_{n, m}(1)=r_{n}, n=1,2, \ldots$ as an initial conditions. For intervals $D_{v, \epsilon}, v=0,1, \ldots, m-1$ the set of eigenvalues are the solutions of the dual equations with initial conditions $u_{n, v}\left(x_{v+1}\right)=\lim _{x \rightarrow x_{v+1}} u_{n,(v+1)}(x)$ and $r_{n, v}\left(x_{v+1}\right)=\lim _{x \rightarrow x_{v+1}} r_{n,(v+1)}(x)$ where $v=1,2, \ldots, m-1, n=1,2, \ldots$

\section{References}

[1] W. Eberhard, G. Freiling and A. Schneider. The distribution of the eigenvalues for second order eigenvalue problems in the presence of an arbitrary number of turning points. Results Math., 21(2):24-41, 1992.

[2] W. Eberhard, G. Freiling and A. Schneider. Connection formulae for secondorder differential equations with a complex parameter and having an arbitrary number of turning points. Math. Nachr., 165(1):205-229, 1994.

http://dx.doi.org/10.1002/mana.19941650114.

[3] G. Freiling and V. Yurko. Inverse problems for differential equations with turning points. Inverse Problems, 13(5):1247-1263, 1997.

http://dx.doi.org/10.1088/0266-5611/13/5/010.

[4] G. Freiling and V. Yurko. Inverse Sturm-Liouville Problems and Their Applications. Nova Sciences, Huntington, New York, 2001.

[5] A.L. Goldenveizer, V.B. Lidsky and P.E. Tovstik. Free Vibration of Thin Elastic Shells. Nauka, Moscow, 1979.

[6] S.G. Halvorsen. A function theoretic property of solutions of the equation $x^{\prime \prime}+$ $(\lambda w-q) x=0$. Quart. J. Math. Oxford, 38(2):73-76, 1987. http://dx.doi.org/10.1093/qmath/38.1.73.

[7] A. Jodayree Akbarfam. Higher-Order Eigenvalue Asymptotics for SturmLiouville Problems with One Simple Turning Point. University of Ottawa, Canada, 1989. PhD Thesis 
[8] A. Jodayree Akbarfam and A.B. Mingarelli. The canonical product of the solution of the Sturm-Liouville equation in one turning point case. Can. Appl. Math. Q., 8(4):305-320, 2000. http://dx.doi.org/10.1216/camq/1032375138.

[9] A. Jodayree Akbarfam and A.B. Mingarelli. Higher order asymptotic distribution of the eigenvalues of non-definite Sturm-Liouville problems with one turning point. J. Comput. Appl. Math., 149(2):423-437, 2002.

http://dx.doi.org/10.1016/s0377-0427(02)00487-9.

[10] A. Jodayree Akbarfam and A.B. Mingarelli. Duality for an indefinite inverse Sturm-Liouville problem. J. Math. Anal. Appl., 312(2):435-463, 2005. http://dx.doi.org/10.1016/j.jmaa.2005.03.096.

[11] A. Jodayree Akbarfam and E. Pourreza. A research note on the second order differential equation. Int. J. Appl. Math., 1:849-862, 1999.

http://dx.doi.org/10.1109/DD.1999.816185.

[12] B.J. Levin. Distribution of Zeros of Entire Functions. American Mathematical Society, 1964.

[13] B.M. Levitan and M.G. Gasymov. Determination of a differential equation by two of its spectra. Russian Math. Surveys, 19(2):1-63, 1964. http://dx.doi.org/10.1070/RM1964v019n02ABEH001145.

[14] B.M. Levitan and M.G. Gasymov. Inverse spectral indefinite problem for the equation $y^{\prime \prime}+\lambda p(x) y=0$ on an interval. Funct. Anal. Appl., 19(2):146-148, 1964. http://dx.doi.org/10.1007/BF01078029.

[15] H.R. Marasi. Asymptotic form and infinite product representation of solution of second-order initial value problem with a complex parameter and having a finite number of turning points. J. Contemp. Math. Anal., 46(4):212-226, 2011. http://dx.doi.org/10.3103/s1068362311040030.

[16] H.R. Marasi and A. Jodayree Akbarfam. On the canonical solution of indefinite problem with m turning points of even order. J. Math. Anal. Appl., 332(2):10711086, 2007. http://dx.doi.org/10.1016/j.jmaa.2006.10.049.

[17] V.A. Marchenko. Certain questions in the theory of differential operators of second order. Dokl. Akad. Nauk. SSSR, 72(21):457-460, 1950. In Russian

[18] J. McHugh. An historical survey of ordinary linear differential equations with a large parameter and turning points. Arch. Hist. Exact Sci., 7(4):277-324, 1994. http://dx.doi.org/10.1007/BF00328046.

[19] A.B. Mingarelli. A survey of the regular weighted Sturm-Liouville problemthe non-definite case. Appl. Diff. Equations, pp. 109-137, 1986. Workshop on Applied Diff. Equations. Beijing, China, World Scientific, Philadelphia, 1986

[20] W.A. Pranger. A formula for the mass density of vibrating string in terms of the trace. J. Math. Anal. Appl., 141(2):399-404, 1989. http://dx.doi.org/10.1016/0022-247X(89)90185-6.

[21] E. Trubowitz. Inverse Spectral Theory. Academic Press, New York, 1987.

[22] W. Wasow. Linear Turning point Theory. Springer, Berlin, 1985. 